\title{
Long-term cycling costs in short-term unit commitment models
}

\author{
Kenneth Van den Bergh, Thomas Legon, Erik Delarue and William D'haeseleer ${ }^{1}$ \\ KU Leuven, Celestijnenlaan 300, 3001 Leuven, Belgium \\ ${ }^{1}$ william.dhaeseleer@kuleuven.be
}

\begin{abstract}
Cycling of conventional power plants is becoming increasingly important in an electricity system with a large penetration of intermittent renewables. Power plant cycling entails short-term costs, e.g., additional fuel costs during start-up, and long-term costs, e.g., additional maintenance costs. Power plant operators should take long-term cycling costs into account when making short-term scheduling decisions, in order to reduce total generation costs. This paper presents a new approach to consider long-term start-up costs in a short-term unit commitment model. The approach is based on an iterative procedure, in which consecutively a unit commitment model is solved and the correct total start-up cost is recalculated. This new approach, referred to as the Cost Redistribution Unit Commitment (CRUC), is applied to a real-life case study based on the 2014 German electricity system. The performance of the CRUC model, in terms of generation costs and computational tractability, is compared with existing start-up cost formulations in the literature. The simulation results show that, for the considered case study, the CRUC model outperforms the existing formulations in terms of generation costs, but requires a longer run time.
\end{abstract}

Index Terms-Power plant cycling, start-up costs, long-term cycling cost, unit commitment.

\section{INTRODUCTION}

Cycling is defined as changing the power output of power plants by ramping-up, ramping-down, starting-up or shuttingdown. Power plants cycle in order to follow load variations in time, and as such maintain the system balance [1].

Historically, electricity generation portfolios have been divided in power plants that barely cycle, i.e., base-load units such as nuclear power plants, and power plants that cycle on a regular basis, i.e., mid- and peak-load units such as gasfired units. However, the type of power plants that cycle and the amount of cycling that they undergo is changing due to the introduction of intermittent renewables in the electricity system. Intermittent renewables, such as solar photovoltaics and wind energy, are variable in time. As a result, the residual load (i.e., the original load minus renewables generation) that has to be followed by conventional generation units is more variable [2]. Therefore, intermittent renewable generation results in increased conventional power plants cycling [3-5].

Cycling entails a cost for the power plant operator. One can distinguish between short-term cycling costs and long-term cycling costs. Short-term cycling costs refer to costs that are directly visible when cycling a power plant, such as additional fuel costs when starting-up a power plant. Long-term cycling costs become only visible after the cycling operation, and are the result of internal damage due to wear and tear [6].
Long-term cycling costs refer to, amongst others, additional maintenance costs. The long-term impact of cycling operations implies that the decision to start-up a power plant today can impact the costs that a power plant operator faces tomorrow. Considerable cost savings can be obtained when total cycling costs, i.e., both short-term and long-term, are taken properly into account in generation scheduling models, especially in a system with a large share of intermittent renewables [7]. It is however not straightforward to take long-term cycling costs into account in short-term electricity generation models such as unit commitment models.

This paper presents a new approach to implement long-term cycling costs in a unit commitment model. More precisely, the focus is on start-up costs. The approach is based on an iterative procedure, solving first a unit commitment model, followed by the calculation of the correct start-up costs after which the unit commitment model is rerun with the updated start-up costs. This approach is referred to as the Cost Redistribution Unit Commitment (CRUC) model. This model is applied to a reallife case study based on the 2014 German electricity system. The performance of the CRUC model is compared to other start-up cost formulations in the literature.

The paper continues as follows. Section II presents the startup cost curve and discusses different start-up cost formulations in unit commitment models. Section III presents the unit commitment model developed for this study, together with the case study. Section IV presents the simulation results and section V concludes.

\section{START-UP COSTS}

In this paper, we aim to better represent the total startup cost (including short-term and long-term costs) in shortterm electricity generation models such as unit commitment models. The first subsection presents the start-up cost curve and the second subsection gives an overview of start-up cost formulations in unit commitment modeling.

\section{A. Start-up cost curve}

The start-up cost curve gives the total cost per start-up (in $€ /$ start-up) as a function of the start-up index (e.g., the cost of the 5th start-up can be read from the start-up cost curve). There is no consensus in the academic literature on how the start-up cost curve looks like. In this paper, a linearly increasing startup cost curve is assumed (see Fig. 1). This start-up cost curve 


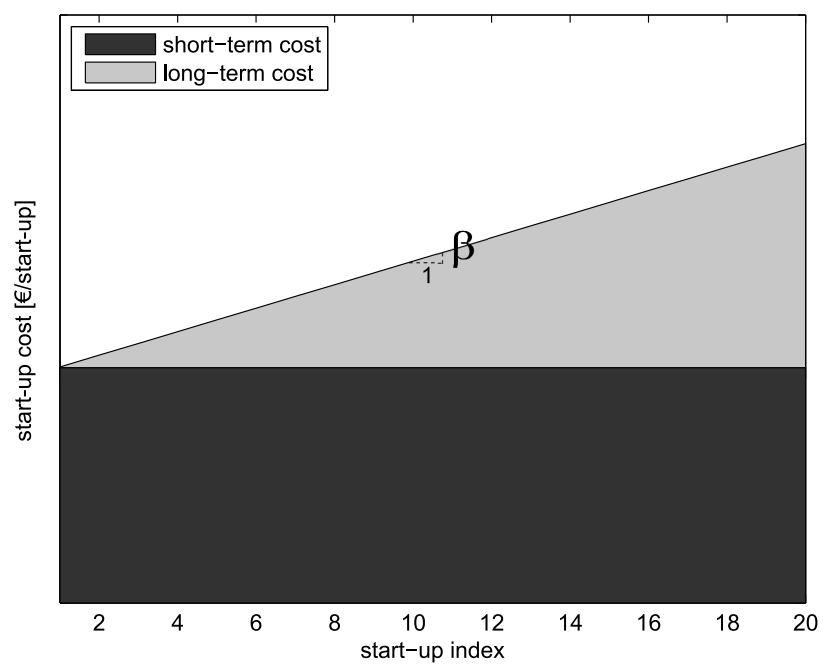

Fig. 1. Illustrative start-up cost curve, with linearly increasing start-up costs ( $\beta$ gives the slope of the curve), consisting of constant short-term start-up costs and increasing long-term start-up costs.

is an input for the rest of the analysis. Note that the start-up cost curve can also be non-linear.

The total start-up cost can be split up in a short-term cost and a long-term cost. The short-term cost is incurred at the moment of cycling and is easy to quantify. The short-term start-up cost includes fuel costs, $\mathrm{CO}_{2}$ emission costs and the cost of auxiliary services to start-up a power plant. This shortterm cost is the same for every start-up and can be read from Fig. 1 as the start-up cost for the first start-up. The long-term cost is incurred after the moment of cycling and is the result of damage accumulation within the power plant. The long-term cost consist of additional capital and maintenance costs, the costs related to a decreasing efficiency and the opportunity cost of not generating electricity during additional outages [8]. The long-term cost is more difficult to quantify, since the damage accumulation within the power plant is a complex and stochastic process. It is assumed in this paper that the long-term cost per start-up increases with the number of startups. The long-term cost related to a certain start-up can be read from Fig. 1 as the start-up cost difference between that start-up and the first start-up.

Extensive literature exists on the link between power plant cycling and damage accumulation of its components. The thermal and pressure changes that occur within a power plant during start-up result in several damage mechanisms which are often referred to with the general term wear and tear. Damage mechanisms that play a role are, amongst others, creep, fatigue, erosion and corrosion. The reader is referred to [9-16] for more information on the link between power plant cycling and component damage accumulation.

\section{B. Start-up costs in unit commitment models}

The most correct way to include start-up costs in unit commitment models is to take the full start-up cost curve into account and solve the unit commitment model for a long period (e.g., a year) at once. As such, the long-term impact of cycling can be properly taken into account in the unit commitment decision. However, it is computationally infeasible to do this for an electricity system of realistic size. Due to computational limitations, the unit commitment model is mostly solved for shorter time horizons such as a day or a week. A longer time horizon (e.g., a year) is then solved by solving sequentially shorter time periods (e.g., days or weeks). In such a sequential approach, it is not straightforward to take the long-term start-up costs into account, since only the next day or week is considered. This results in suboptimal generation scheduling.

In the literature, one finds three different ways of implementing start-up costs in unit commitment models: (1) start-up costs are not considered, (2) a static start-up cost is imposed or (3) a dynamic start-up cost is imposed. In this paper, the authors present a fourth approach for implementing start-up costs, which better captures the long-term start-up costs.

1) Zero start-up costs (ZSUC): In a ZSUC model, start-up costs are neglected and not taken into account in the unit commitment model. Although this is easy to implement, the true total system cost will be underestimated by neglecting start-up costs, leading to suboptimal unit commitment decisions.

2) Static start-up costs (SSUC): In a SSUC model, a timeconstant start-up cost is imposed to the unit commitment model:

$$
c_{i, t}^{\text {start }}=S U C_{i} v_{i, t} \quad \forall i, t
$$

with $c_{i, t}^{\text {start }}$ the start-up cost of power plant $i$ at time step $t$ in $€, S U C_{i}$ the static start-up cost of power plant $i$ in $€ /$ startup and $v_{i, t}$ the start-up status of power plant $i$ at time step $t$. In this paper, the short-term start-up cost is taken as the static start-up cost $\left(S U C_{i}=S T C_{i}\right.$ with $S T C_{i}$ the short-term start-up cost of power plant $i$ in $€ /$ start-up).

3) Dynamic start-up costs (DSUC): In a DSUC model, a time-variable start-up cost is imposed (i.e., the start-up cost depends on the start-up index). Dynamic start-up costs were introduced by Troy et al [17].

$$
\begin{gathered}
c_{i, t}^{\text {start }}=S T C_{i} v_{i, t}+L T C_{i, t} \quad \forall i, t \\
0 \leq L T C_{i, t} \geq\left(n_{i, t}^{\text {start }}-1\right) \beta_{i}-M\left(1-v_{i, t}\right) \quad \forall i, t \\
n_{i, t}^{\text {start }} \geq n_{i,(t-1)}^{\text {start }}+v_{i, t} \quad \forall i, t
\end{gathered}
$$

with $L T C_{i, t}$ the time-variable long-term start-up cost of power plant $i$ at time step $t$ in $€, n_{i, t}^{\text {start }}$ the start-up index of power plant $i$ at time step $t, \beta_{i}$ the slope of the start-up cost curve of power plant $i$ in $€ /$ startup and $M$ a big number. Eq. (2) defines the start-up cost as the sum of the short-term start-up cost and the long-term start-up cost. Eq. (3) sets the long-term start-up cost to zero when no start-up occurs and to the correct long-term start-up cost when a start-up occurs. Eq. (4) defines the start-up index. The parameter $\beta_{i}$ is assumed to be $5 \%$ of the short-term start-up cost of power plant $i$. 
(1) Solve for initial solution

(2) New start-up cost calculation based on start-ups in prev. step

(3) Solve sequential UC-model with new cost information

(4) Is the start-up quantity per power plant constant? yes

(5) Solution found

Fig. 2. The flow chart illustrates the iterative approach of the cost redistribution unit commitment model.

4) Cost redistribution unit commitment (CRUC): The CRUC model, a new approach proposed by the authors, consists of an iterative procedure, summarized on Fig. 2. First, the unit commitment model is solved for a long time horizon by sequentially solving shorter optimizations. In this first step, the short-term start-up cost is imposed as a static start-up cost (see Eq. (1)). Second, a new static start-up cost can be calculated, based on the simulation results from the previous steps, by dividing the total incurred start-up cost (i.e., the integral of the start-up cost curve, running from zero to the total number of start-ups) with the number of start-ups:

$$
S U C_{i}^{k}=\frac{\left(S T C_{i}+0.5 \beta_{i} \sum_{t} v_{i, t}\right) \sum_{t} v_{i, t}}{\sum_{t} v_{i, t}} \quad \forall i
$$

with $S U C_{i}^{k}$ the static start-up cost of power plant $i$ in iteration $k$. Third, the unit commitment model is rerun with the updated static start-up cost. In a fourth step, convergence is checked. If the number of start-ups of each power plant remains constant in two consecutive unit commitment runs, the optimal solution is found. If not, the iterative loop continues. In short, the CRUC model comes down to redistributing the aggregated total start-up costs incurred by a power plant equally over every start-up.

\section{Methodology}

A unit commitment model is deployed for this study. The different start-up cost formulations, discussed in the previous section, are implemented in this unit commitment model, and the unit commitment model is applied to a Germany-based case study. This section presents the unit commitment model and the case study.

\section{A. Model description}

A unit commitment model is an operational scheduling model of the electricity generation system. In this paper, a full year is considered with an hourly time step. The objective function of the unit commitment model is to minimize the total operational system cost, consisting of generation cost (i.e., fuel costs) and start-up costs:

$$
\min _{p_{i, t}, z_{i, t}, v_{i, t}, w_{i, t}} \sum_{i, t}\left(M C_{i} p_{i, t}+c_{i, t}^{s t a r t}\right)
$$

with $M C_{i}$ the marginal generation cost of power plant $i$ in $€ / \mathrm{MWh}$ and $p_{i, t}$ the power output of power plant $i$ at time step $t$ in MW.

The objective function is subject to the market clearing condition (Eq. (7)), power plant generation limits (Eq. (8)), ramping limits (Eqs. (9)-(10)), minimum up and down time limits (Eqs. (11)-(12)), the binary logic constraint (Eq. (13)) and binary constraints (Eq. (14)).

$$
\begin{gathered}
\sum_{i} p_{i, t}=R D_{t} \quad \forall t \\
P_{i}^{\text {min }} z_{i, t} \leq p_{i, t} \leq P_{i}^{\max } z_{i, t} \quad \forall i, t \\
p_{i, t}-p_{i, t-1} \leq R U_{i} z_{i, t-1}+S U_{i} v_{i, t} \quad \forall i, t \\
p_{i, t-1}-p_{i, t} \leq R D_{i} z_{i, t}+S D_{i} w_{i, t} \quad \forall i, t \\
z_{i, t} \geq \sum_{t^{\prime}=t+1-M U T_{i}}^{t} v_{i, t^{\prime}} \quad \forall i, t \\
\sum_{t^{\prime}=t+1-M D T_{i}}^{t} w_{i, t^{\prime}} \quad \forall i, t \\
v_{i, t}-w_{i, t}=z_{i, t}-z_{i, t-1} \quad \forall i, t \\
v_{i, t}, w_{i, t}, z_{i, t} \in\{0,1\} \quad \forall i, t
\end{gathered}
$$

with $R D_{t}$ the residual load (i.e., original load minus renewables generation) at time step $t$ in $\mathrm{MW}, P_{i}^{\text {min }}$ the minimum output of power plant $i$ in $\mathrm{MW}, P_{i}^{\max }$ the maximum output of power plant $i$ in $\mathrm{MW}, R U_{i}$ and $R D_{i}$ the maximum rampingup and ramping-down rate of power plant $i$ in $\mathrm{MW} / \mathrm{h}, S U_{i}$ and $S D_{i}$ the maximum starting-up and shutting-down rate of power plant $i$ in $\mathrm{MW} / \mathrm{h}, M U T_{i}$ and $M D T_{i}$ the minimum up and down times of power plant $i$ in $\mathrm{h}, z_{i, t}$ the on/off-state of power plant $i$ at time step $t, v_{i, t}$ the start-up state of power plant $i$ at time step $t$ and $w_{i, t}$ the shut-down state of power plant $i$ at time step $t$.

The unit commitment model is formulated as a mixedinteger linear program in GAMS 24.4 and solved by CPLEX 12.6 with a stopping tolerance of $0.001 \%$. No network constraints are considered in this model. Simulations were run on an Intel@ Core $^{\mathrm{TM}}$ i7-2600 Quad core, clocked at $3.8 \mathrm{GHz}$.

\section{B. System description}

The case study is based on the 2014 German electricity system. A full year (i.e., 8760 hours) is considered with an hourly time step.

Table I gives an overview of the conventional generation portfolio, containing all operational power plants with an electric power output larger than $100 \mathrm{MW}$ [18]. The generation portfolio consists of 230 power plants with an aggregated capacity of $86.5 \mathrm{GW}$. Power plant efficiencies are assumed to depend on the commissioning year of the power plant. ${ }^{1}$ The following fuel prices are used: $1.7 € / \mathrm{MWh}_{\mathrm{th}}$ for uranium,

\footnotetext{
${ }^{1}$ The highest rated efficiency is allocated to units commissioned or retrofitted after 2000, the middle-most to units commissioned between 1986 and 2000, and the lowest to units commissioned before 1986.
} 
TABLE I

OVERVIEW OF THE CONVENTIONAL GENERATION PORTFOLIO [18] (SPP: STEAM POWER PLANT; CCGT: COMBINED-CYCLE GAS TURBINES; OCGT: OPEN-CYCLE GAS TURBINES; $\eta$ : RATED EFFICIENCY; STC: SHORT-TERM START-UP COST).

\begin{tabular}{|l|rrrr|} 
& \# units & $\begin{array}{r}\eta \\
\text { capacity }\end{array}$ & $\begin{array}{r}S T C \\
{[\%]}\end{array}$ & $\begin{array}{r}S T / \mathrm{MW} / \text { start] } \\
{[\text { [GW }}\end{array}$ \\
\hline Nuclear & 8 & 11.4 & 33 & 300 \\
SPP-coal & 67 & 26.2 & $35-40-46$ & 25 \\
SPP-lignite & 43 & 22.3 & $35-40-46$ & 28 \\
SPP-gas & 8 & 2.5 & $36-41$ & 33 \\
CCGT-gas & 72 & 19.1 & $48-58$ & 5 \\
OCGT-gas & 32 & 5.2 & $35-42$ & 2.4 \\
\hline
\end{tabular}

TABLE II

TECHNICAL POWER PLANT PARAMETERS OF THE CONVENTIONAL GENERATION PORTFOLIO [20] (SPP: STEAM POWER PLANTS; CCGT: COMBINED-CYCLE GAS TURBINES; OCGT: OPEN-CYCLE GAS TURBINES).

\begin{tabular}{|l|rrr|}
\hline & $P^{\min }$ & $M U T, M D T$ & $R U, R D, S U, S D$ \\
& {$\left[\% P^{\max }\right]$} & {$[\mathrm{h}]$} & {$\left[\% P^{\max } / h\right]$} \\
\hline Nuclear & 50 & 24 & 70 \\
SPP-coal & 40 & 6 & 70 \\
SPP-lignite & 40 & 24 & 70 \\
SPP-gas & 40 & 5 & 70 \\
CCGT & 35 & 3 & 100 \\
OCGT & 25 & 1 & 100 \\
\hline
\end{tabular}

4.0 $€ / \mathrm{MWh}_{\text {th }}$ for lignite, $7.5 € / \mathrm{MWh}_{\text {th }}$ for coal and 21.1 $€ / \mathrm{MWh}_{\text {th }}$ for natural gas [19]. The $\mathrm{CO}_{2}$ emission cost is assumed to be zero. Table II gives an overview of the technical power plant parameters used in this study [20].

The hourly residual load time series is composed by deducting historical renewable generation from the original load. The original load time series originates from ENTSO-E, and is corrected for import/export with neighbouring countries and pumped storage consumption/generation [21]. Renewables generation for wind, solar photovoltaics, run-of river and biomass are taken from the four German TSOs [22-25]. Annual residual load is $316 \mathrm{TWh}$, with a peak of $68.5 \mathrm{GW}$.

\section{RESUlts}

Simulations of the real-life case study are run with the four different start-up cost formulations presented in section II.B. The simulation results are compared in terms of cost optimality and run times.

Table III summarizes the simulation results. It is important to mention that the costs presented in this table follow from an ex-post calculation of the start-up costs, based on the number of start-ups indicated by the simulation and the start-up cost curve (see Fig. 1). The CRUC model results in the lower total system cost, slightly lower than the DSUC and SSUC model and considerably lower than the ZSUC model. Looking at the start-up costs, a similar trend is visible, with the CRUC model outperforming the other formulations (note however that the unit commitment model minimizes total costs, not start-up costs). When comparing the DSUC formulation with the CRUC formulation, total system costs decrease with 1.7 $\mathrm{M} €$ per year or $0.05 \%$ when using the CRUC formulation.
TABLE III

OVERVIEW OF SIMULATION RESULTS (YEARLY AGGREGATED). THE CRUC GIVES MORE OPTIMAL RESULTS IN TERMS OF SYSTEM COSTS THAN THE OTHER START-UP COST FORMULATIONS, BUT REQUIRES A LONGER RUN TIME.

\begin{tabular}{|c|rrrr|}
\hline & ZSUC & SSUC & DSUC & CRUC \\
\hline Total costs [M $€$ ] & $4,105.4$ & $3,522.2$ & $3,511.0$ & $3,509.3$ \\
relative to SSUC & $16.56 \%$ & & $-3.18 \%$ & $-3.66 \%$ \\
Start-up costs [M€] & 674.4 & 70.88 & 51.46 & 46.43 \\
relative to SSUC & $851.46 \%$ & & $-27.40 \%$ & $-34.50 \%$ \\
Run time [h] & 1.4 & 1.6 & 3 & 39 \\
\hline
\end{tabular}

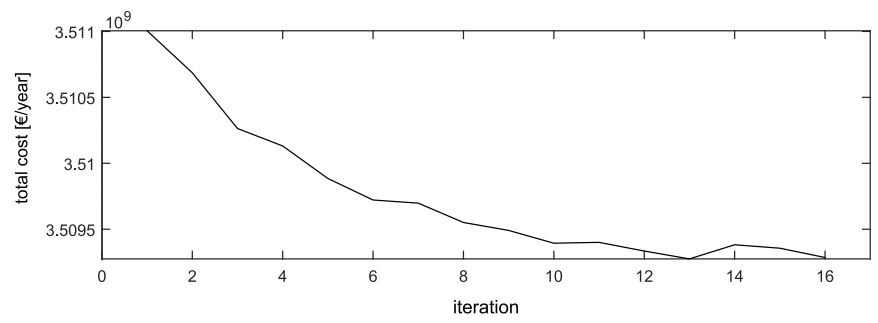

Fig. 3. Evolution of the total cost in consecutive iterations (CRUC model).

Note that the start-up cost is a rather small part of the total system cost (between $1 \%$ and $16 \%$ in the considered formulations), so the impact of improving start-up costs on total systems costs is also rather limited. However, one can expect that the importance of start-up costs increase with a higher penetration of intermittent renewables.

In terms of run times, Table III shows that the CRUC requires a 13 times longer run time than the DSUC formulation. The gain in system cost savings hence comes at a computational price.

Fig. 3 illustrates the evolution of total costs in consecutive CRUC iterations. It is observed that total costs decrease during the first 13 iterations, but that the iterative procedure starts oscillating between different solutions afterwards. Therefore an additional stopping criterion is required (besides the absolute cost difference between to subsequent iterations), stopping the iterative procedure when the total cost of the next iteration is higher. It is shown in Fig. 3 that this occurs at iteration 13-14. Note also that as of the second iteration, the CRUC model results in lower total costs than the DSUC model.

The reason that the CRUC is outperforming the other formulations in terms of system costs is that the iterative procedure allows to consider long time horizons (e.g., a year). As such, the long-term impact of cycling can be incorporated in the short-term unit commitment model. The DSUC model does take into account the correct start-up cost curve with increasing start-up costs as a function of the number of startups, but only considers a short time horizon and hence doesn't capture the long-term impact of cycling. As such, the impact of cycling today on the cycling costs in the future is not covered by the DSUC model. 


\section{CONClusion}

This paper proposes a new start-up cost formulation for unit commitment models. Start-up costs are becoming increasingly important in a system with a large penetration of intermittent renewables. Start-up costs can be split up in short-term costs, i.e., costs that occur at the moment of cycling such as additional fuel costs, and long-term costs, i.e., costs that occur after the moment of cycling such as additional maintenance costs. It is challenging to take long-term cycling costs into account in short-term generation scheduling models such as unit commitment models.

In order to better represent these long-term costs in unit commitment models, the paper proposes a new iterative procedure. The procedure solves consecutively a unit commitment model and calculates the correct total start-up cost. This new approach is referred to as the Cost Redistribution Unit Commitment (CRUC).

The Cost Redistribution Unit Commitment (CRUC) is compared with three existing start-up cost formulations, for a case study of a 2014 Germany-based electricity system. The simulations show that, for the considered case study, the CRUC model outperforms the other start-up cost formulations in terms of system costs, but requires longer run times.

Future work consists of extending the presented deterministic CRUC model to a stochastic model. In the CRUC model, a long time horizon is considered (e.g., a year), but load and renewables time series are uncertain for this time horizon. A stochastic model can take this uncertainty into account.

\section{REFERENCES}

[1] P. Keatley, A. Shibli, and N. Hewitt. Estimating power plant start costs in cyclic operation. Applied Energy, 111: 550-557, 2013.

[2] H. Holttinen. Impact of hourly wind power variations on the system operation in the Nordic countries. Wind Energy, 8(2):197-218, 2005.

[3] N. Troy, E. Denny, and M. O’Malley. Base-load cycling on a system with significant wind penetration. IEEE Transactions on Power Systems, 25(2):1088-1097, 2010.

[4] I. Perez-Arriaga, C. Batlle, et al. Impacts of intermittent renewables on electricity generation system operation. Economics of Energy \& Environmental Policy, 1(2), 2012.

[5] S. Lefton, N. Kumar, D. Hilleman, and D. Agan. The increased cost of cycling operations at combined cycle power plants. Intertek APTECH, November, 2012.

[6] S. Lefton and P. Besuner. The cost of cycling coal fired power plants. Coal Power Magazine, pages 16-20, 2006.

[7] N. Kumar, P. Besuner, S. Lefton, D. Agan, and D. Hilleman. Power plant cycling costs. Technical report.

[8] K. Van den Bergh and E. Delarue. Cycling of conventional power plants: technical limits and actual costs. Energy Conversion and Management, 97:70-77, 2015.

[9] T. Elliott, K. Chen, and R. Swanekamp. Standard Handbook of Powerplant Engineering. McGraw-Hill Education, 1998.
[10] L. Drbal, K. Westra, and P. Boston. Power plant engineering. Springer Science \& Business Media, 2012.

[11] F. Starr. Effects of cyclic operation on advanced energy conversion systems. Materials at high temperatures, 20 (1):27-37, 2003.

[12] S. Holdsworth, E. Mazza, L. Binda, and L. Ripamonti. Development of thermal fatigue damage in $1 \mathrm{CrMoV}$ rotor steel. Nuclear Engineering and Design, 237(24): 2292-2301, 2007.

[13] E. Denny and M. OMalley. The impact of carbon prices on generation-cycling costs. Energy Policy, 37(4):12041212, 2009.

[14] T. Seifert and H. Riedel. Mechanism-based thermomechanical fatigue life prediction of cast iron - part i: models. International Journal of Fatigue, 32(8):13581367, 2010.

[15] A. Nagesha, R. Kannan, P. Parameswaran, R. Sandhya, K. Rao, and V. Singh. A comparative study of isothermal and thermomechanical fatigue on type $316 \mathrm{~L}$ (N) austenitic stainless steel. Materials Science and Engineering: A, 527(21):5969-5975, 2010.

[16] S. Holmström and P. Auerkari. A robust model for creep-fatigue life assessment. Materials Science and Engineering, 559:333-335, 2013.

[17] N. Troy, D. Flynn, M. Milligan, and M. O’Malley. Unit commitment with dynamic cycling costs. IEEE Transactions on Power Systems, 27(4):2196-2205, 2012.

[18] Umwelt Bundesamt. Datenbank Kraftwerke in Deutschland, 2015.

[19] K. Van den Bergh. Unit Commitment Model of the European Electricity Generation System. Working Paper, 2015.

[20] A. Schröder, F. Kunz, J. Meiss, R. Mendelevitch, and C. Von Hirschhausen. Current and prospective costs of electricity generation until 2050. DIW Data Documentation, 2013.

[21] ENTSO-E. Statistical Database. Available at www.entsoe.eu/data/data-portal, 2015.

[22] 50Hertz. Photovoltaic and wind infeed. Available at www.50hertz.com/en/Renewables, 2015.

[23] Amprion. Grid data. Available at www.amprion.net/en/grid-data, 2015.

[24] TenneT. Wind infeed. Available at www.tennettso.de/site/en/Transparency/publications/networkfigures/overview, 2015.

[25] Transnet BW. Wind infeed. Available at www.transnetbw.com/en/key-figures, 2015. 